\title{
A MULTIPLICITY RESULT FOR A SYSTEM OF REAL INTEGRAL EQUATIONS BY USE OF THE NIELSEN NUMBER
}

\author{
ANDREI Yu. BORISOVICH \\ Institute of Mathematics, University of Gdansk \\ Wita Stwosza 57, 80-952 Gdańsk, Poland \\ E-mail:matab@univ.gda.pl \\ ZYGFRYD KUCHARSKI \\ Faculty of Technical Physics and Applied Mathematics \\ Technical University of Gdańsk \\ G. Narutowicza 11/12, 80-952 Gdańsk, Poland \\ E-mail: zkuchar@mif.pg.gda.pl \\ WACłAW MARZANTOWICZ \\ Faculty of Mathematics \& Computer Science \\ A. Mickiewicz University of Poznań \\ Matejki 48/49, 60-769 Poznań, Poland \\ E-mail:marzan@amu.edu.pl
}

\begin{abstract}
We prove an existence and multiplicity result for solutions of a nonlinear Urysohn type equation (2.14) by use of the Nielsen and degree theory in an annulus in the function space.

1. Main scheme. Consider a family of nonlinear equations

$$
x=G_{\lambda}(x)
$$

depending continuously on the parameter $\lambda \in[0,1]$, where $G_{\lambda}: X \rightarrow X$ are continuous selfmaps of a Banach space $X$. The homotopy $G_{\lambda}$ is thought of as a deformation of $G_{1}(x)=x$ to a simpler equation $G_{0}(x)=x$. We look for some open path-connected subset or ANR $D \subset X$, which is invariant with respect to the maps $G_{\lambda}$, i.e.
\end{abstract}

$$
G_{\lambda}(D) \subset D, \quad \lambda \in[0,1]
$$

1991 Mathematics Subject Classification: Primary 55M20; Secondary 57N05.

Research supported by KBN grant 2-P03A-080-08.

The paper is in final form and no version of it will be published elsewhere. 
and then we restrict our considerations to $D$ under the following assumptions:

(A) The map $\widehat{G}: X \times[0,1] \rightarrow X \times[0,1]$, defined by $\widehat{G}(x, \lambda)=\left(G_{\lambda}(x), \lambda\right)$, is completely continuous.

(B) The set $\operatorname{Fix}(\widehat{G}, D \times[0,1])$ of fixed points of $\widehat{G}$ which belong to $D \times[0,1]$ is a compact subset of $X \times[0,1]$.

(C) The equation $x=G_{0}(x)$ has precisely $n$ solutions $\left\{x_{1}^{0}, \ldots, x_{n}^{0}\right\}$ in $D$ and there exist open nieghborhoods $U_{j}^{0}(j=1, \ldots, n)$ of $x_{j}^{0}$ such that

$$
\begin{array}{ll}
U_{i}^{0} \cap U_{j}^{0}=\emptyset, & i \neq j, \\
\operatorname{deg}\left(I-G_{0}, U_{j}^{0}, 0\right) \neq 0, & j=1, \ldots, n,
\end{array}
$$

where $I: X \rightarrow X$ is the identity map.

The following result can be obtained using the Nielsen fixed point theory.

TheOREm 1.1. Assume that the conditions $(\mathrm{A})-(\mathrm{C})$ are satisfied. If the fixed points $x_{1}^{0}, \ldots, x_{n}^{0}$ of $G_{0}$ are in different Nielsen classes, then for each $\lambda \in[0,1]$ the equation $x=G_{\lambda}(x)$ has at least $n$ solutions, which belong to different Nielsen classes of $G_{\lambda}$.

Recall that two fixed points $x_{i}^{0}$ and $x_{j}^{0}$ belong to the same Nielsen class if there exists a continuous path $w$ joining $x_{i}^{0}$ and $x_{j}^{0}$ such that $w$ and its image $G_{0}(w)$ are homotopic in $D$ rel end points. The Nielsen class $\{x\}$ is called essential if there exists an open neighbourhood $U$ such that

$$
\operatorname{Fix}\left(G_{0}, D\right) \cap U=\{x\}, \quad \operatorname{deg}\left(I-G_{0}, U, 0\right) \neq 0 .
$$

The number $\mathbf{N}\left(G_{0}, D\right)$ of essential classes is called the Nielsen number. It is a homotopy invariant, i.e. if $G_{1}$ is homotopic to $G_{0}$ by a homotopy $G_{\lambda}: D \rightarrow D$ which satisfies assumptions (A)-(B), then $\mathbf{N}\left(G_{0}, D\right)=\mathbf{N}\left(G_{1}, D\right)$. Such a homotopy $G_{\lambda}: D \rightarrow D$ is called admissible. In our situation, the fixed points $x_{1}^{0}, \ldots, x_{n}^{0}$ by $(\mathrm{C})$ belong to different essential Nielsen classes and

$$
\mathbf{N}\left(G_{\lambda}, D\right)=n
$$

for each $\lambda \in[0,1]$. For more details about Nielsen classes see $[\mathrm{K}],[\mathrm{J}],[\mathrm{Br} 3]$.

REMARK 1.1. If $D$ is simply-connected, then all fixed points in $D$ belong to the same Nielsen class. Theorem 1.1 gives a multiplicity result only for a non-simply-connected domain $D$.

There are very few papers employing the Nielsen theory to nonlinear problems $([\mathrm{Br} 2]$, $[\mathrm{Br} 3],[\mathrm{F}],[\mathrm{BKM} 1])$.

2. Systems of equations. In this note we study a class of nonlinear systems of integral equations of Urysohn type. Using the Nielsen number we show that the discussed system has at least two non-zero solutions. The form of the integral kernel yields an a priori estimate which guarantees that the linear deformation of the original map preserves the annulus. 
We will work in the Banach space $X=C[0,1] \times C[0,1]$ of pairs of continuous functions with the norm

$$
x=(u, v), \quad\|x\|=\bar{u}+\bar{v},
$$

where $\bar{u}=\max |u(t)|$ and $\bar{v}=\max |v(t)|$.

In $C[0,1]$ we consider two closed cones of positive and of negative continuous functions, respectively:

$$
C^{+}[0,1]=\{u(t): u(t) \geqslant 0\}, \quad C^{-}[0,1]=\{u(t): u(t) \leqslant 0\} .
$$

We will also use the set

$$
C^{ \pm}[0,1]=C^{+}[0,1] \cup C^{-}[0,1]
$$

Definition 2.1. By the annulus in the Banach space $X=C[0,1] \times C[0,1]$ we shall understand the set

$$
A_{c}=C^{ \pm}[0,1] \times C^{ \pm}[0,1]-\{(0,0)\} .
$$

Lemma 2.1. The set $A_{c}$ is a path-connected $A N R$ and the fundamental group of $A_{c}$ is isomorphic to the group of integer numbers, i.e.

$$
\pi_{1}\left(A_{c}\right) \simeq \mathbb{Z}
$$

Proof. Consider the two-dimensional subspace of pairs of constant functions in $X$

$$
E^{2}=\left\{\left(c_{1}, c_{2}\right): c_{i} \in \mathbb{R}\right\} .
$$

We denote by $E_{0}^{2}$ this plane with the point $(0,0)$ deleted. Notice that $E_{0}^{2} \subset A_{c}$. Moreover, we have the deformation retraction $\rho: A_{c} \times[0,1] \rightarrow A_{c}$ defined by the formula

$$
\rho(u, v, \lambda)=(\lambda u+(1-\lambda) \operatorname{sign} u \cdot \bar{u}, \lambda v+(1-\lambda) \operatorname{sign} v \cdot \bar{v}),
$$

such that

$$
\rho\left(A_{c}, 1\right)=A_{c}, \quad \rho\left(A_{c}, 0\right)=E_{0}^{2} .
$$

Therefore, we have

$$
\pi_{1}\left(A_{c}\right)=\pi_{1}\left(E_{0}^{2}\right)=\pi_{1}\left(S^{1}\right)=\mathbb{Z}
$$

Next, consider a function $g: \mathbb{R}^{2} \rightarrow \mathbb{R}^{2}$ defined by

$$
g\left(c_{1}, c_{2}\right)=\left(\delta_{1} c_{2}^{\beta}, \delta_{2} c_{1}^{\alpha}\right),
$$

where $\alpha$ and $\beta$ are positive rational numbers, $m$ and $n$ are relatively prime, $c^{\frac{n}{m}}=(\operatorname{sign} c$. $\left.|c|^{\frac{1}{m}}\right)^{n}$ by definition, and $\delta_{1}, \delta_{2}$ are nonzero. Note that $g\left(\mathbb{R}_{0}^{2}\right) \subset \mathbb{R}_{0}^{2}$, where $\mathbb{R}_{0}^{2}$ is $\mathbb{R}^{2}$ with the point $(0,0)$ deleted.

LEMMA 2.2. For given positive rational numbers $\alpha=\frac{n_{1}}{m_{1}}, \beta=\frac{n_{2}}{m_{2}}$ such that $\alpha \cdot \beta \neq 1$, and for $\delta_{1}, \delta_{2} \in\{-1,+1\}$ define the continuous map $g: \mathbb{R}_{0}^{2} \rightarrow \mathbb{R}_{0}^{2}$ by (2.10). Then the fixed point set of $g$ is compact, and the degree of $g$ is given by the formula

$$
\operatorname{deg}(g)=-\delta_{1} \delta_{2}\left(\frac{1-(-1)^{n_{1}}}{2}\right)\left(\frac{1-(-1)^{n_{2}}}{2}\right),
$$

and consequently the Nielsen number

$$
\mathbf{N}\left(g, \mathbb{R}_{0}^{2}\right)=|1-\operatorname{deg}(g)| \in\{0,1,2\} .
$$


Proof. The first part follows from the fact that the degree is multiplicative. Since for $\alpha=\frac{n}{m}$ we have $\operatorname{deg}\left(x^{\alpha}\right)=0$ or 1 depending on whether $n$ is even or odd, the second part of the statement is a property of the Nielsen number of a selfmap of $S^{1}$, or equivalently of $\mathbb{R}_{0}^{2}$.

REMARK 2.1. If $\delta_{1}=\delta_{2}$ and both $n_{1}, n_{2}$ are odd numbers, then $\mathbf{N}\left(g, \mathbb{R}_{0}^{2}\right)=2$.

Remark 2.1 has a simple geometrical sense. The fixed points of $g$ are given as solutions of the system

$$
\left(c_{1}, c_{2}\right)=\left(\delta_{1} c_{2}^{\beta}, \delta_{2} c_{1}^{\alpha}\right)
$$

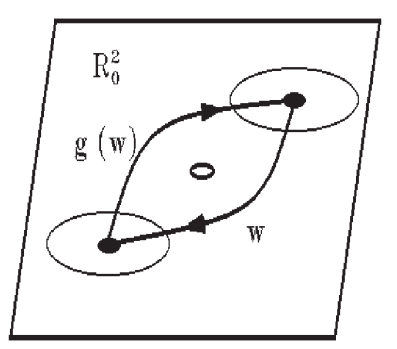

Fig. 1

If $\delta_{1}=\delta_{2}$ and $n_{1}, n_{2}$ are odd, then (2.13) has two solutions:

$$
\begin{array}{lll}
(-1,-1) & \text { and } \quad(+1,+1) & \text { if } \delta_{1}=\delta_{2}=1, \\
(-1,+1) & \text { and } \quad(+1,-1) & \text { if } \delta_{1}=\delta_{2}=-1,
\end{array}
$$

which are different essential Nielsen classes (see Fig. 1).

We are in a position to formulate our main theorem.

Consider the following system of two nonlinear real integral equations:

$$
\left\{\begin{array}{l}
u(t)=\int_{0}^{1} K_{1}(t, s, u(s), v(s)) v^{\beta}(s) d s, \\
v(t)=\int_{0}^{1} K_{2}(t, s, u(s), v(s)) u^{\alpha}(s) d s,
\end{array}\right.
$$

where $\alpha$ and $\beta$ are positive rational numbers, $u^{\frac{n}{m}}=\left(\operatorname{sign} u \cdot|u|^{\frac{1}{m}}\right)^{n}$ by definition. System (2.14) is equivalent to the operator equation $x=G(x)$, where the operator $G: X \rightarrow X$ is defined by the formula

$$
G(u, v)=\left(\int_{0}^{1} K_{1}(\ldots) v^{\beta}(s) d s, \int_{0}^{1} K_{2}(\ldots) u^{\alpha}(s) d s\right),
$$

and hence is a completely continuous.

THEOREM 2.1. Suppose (2.14) satisfies the following assumptions:

1) $K_{i}(t, s, u, v) \in C^{1}\left([0,1]^{2} \times \mathbb{R}^{2}\right)$ for $i=1,2$;

2) $\underline{K} \leqslant\left|K_{i}(t, s, u, v)\right| \leqslant \bar{K}$ for all $(t, s, u, v) \in[0,1]^{2} \times \mathbb{R}^{2}$, where $0<\underline{K} \leqslant 1 \leqslant \bar{K}$;

3) $\alpha=\frac{n_{1}}{m_{1}}, \beta=\frac{n_{2}}{m_{2}} \in \mathbb{Q}_{+}$and $\alpha \beta \neq 1$. 
Then the operator $G: A_{c} \rightarrow A_{c}$ (see (2.15)) is well defined, the set $\operatorname{Fix}\left(G, A_{c}\right)$ is compact, the Nielsen number $\mathbf{N}\left(G, A_{c}\right)$ is well defined and

$$
\mathbf{N}\left(G, A_{c}\right)=\mathbf{N}\left(g, \mathbb{R}_{0}^{2}\right),
$$

where $g: \mathbb{R}_{0}^{2} \rightarrow \mathbb{R}_{0}^{2}$ is the map defined in (2.10) with $\delta_{i}=\operatorname{sign} K_{i}$. Consequently, the system (2.1) has at least 2 non-zero solutions if $\delta_{1}=\delta_{2}$ and $n_{1}, n_{2}$ are odd.

Proof. Deform the system (2.14) to a simpler system

$$
\left\{\begin{array}{l}
u(t)=\int_{0}^{1} \delta_{1} v^{\beta}(s) d s \\
v(t)=\int_{0}^{1} \delta_{2} u^{\alpha}(s) d s
\end{array}\right.
$$

which is equivalent to the operator equation $x=G_{0}(x)$, where $G_{0}: X \rightarrow X$ is defined by

$$
G_{0}(u, v)=\left(\int_{0}^{1} \delta_{1} v^{\beta}(s) d s, \int_{0}^{1} \delta_{2} u^{\alpha}(s) d s\right) .
$$

Consider a linear homotopy $x=G_{\lambda}(x), \lambda \in[0,1]$, connecting $G=G_{1}$ with $G_{0}$, which is defined by

$$
G_{\lambda}=\lambda G_{1}+(1-\lambda) G_{0}
$$

Explicitly, we have the equations

$$
\left\{\begin{array}{l}
u(t)=\int_{0}^{1}\left(\lambda K_{1}(t, s, u(s), v(s))+(1-\lambda) \delta_{1}\right) v^{\beta}(s) d s, \\
v(t)=\int_{0}^{1}\left(\lambda K_{2}(t, s, u(s), v(s))+(1-\lambda) \delta_{2}\right) u^{\alpha}(s) d s,
\end{array}\right.
$$

thus the operator $G_{\lambda}: X \rightarrow X$ is of the form

$$
G_{\lambda}(u, v)=\left(\int_{0}^{1} \widetilde{K}_{1}(t, s, u(s), v(s), \lambda) v^{\beta}(s) d s, \int_{0}^{1} \widetilde{K}_{2}(t, s, u(s), v(s), \lambda) u^{\alpha}(s) d s\right),
$$

where the kernels $\widetilde{K}_{1}, \widetilde{K}_{2}$ are given by the right side of (2.20).

Let us verify conditions (A)-(C) for the family $(2.21)$.

The map $\widehat{G}: X \times[0,1] \rightarrow X \times[0,1]$, defined by $\widehat{G}(x, \lambda)=\left(G_{\lambda}(x), \lambda\right)$, is completely continuous. This follows from the smoothness of $\widetilde{K}_{1}, \widetilde{K}_{2}$ (see the first assumption of Theorem 2.1), from $G_{\lambda}:(C[0,1])^{2} \rightarrow\left(C^{1}[0,1]\right)^{2}$ and from the existence of a completely continuous embedding $i:\left(C^{1}[0,1]\right)^{2} \rightarrow(C[0,1])^{2}$.

The set $A_{c}$ is an ANR in $X$ and

$$
G_{\lambda}\left(A_{c}\right) \subset A_{c}
$$

for each $\lambda \in[0,1]$. This follows from assumption 2 of Theorem 2.1.

For the proof that the set $\operatorname{Fix}\left(\widehat{G}, A_{c} \times[0,1]\right)$ is a compact subset of $X \times[0,1]$ we need the following lemma. 
LEMMA 2.3. Suppose that there exist two constants $0<r<R$ such that for every pair $(x, \lambda) \in A_{c} \times[0,1]$ which satisfies $x=G_{\lambda}(x)$ we have

$$
r \leqslant\|x\| \leqslant R
$$

Then the set $\operatorname{Fix}\left(\widehat{G}, A_{c} \times[0,1]\right)$ is a compact subset in $X \times[0,1]$.

Obviously, the set $\operatorname{Fix}(\widehat{G}, X \times[0,1])$ of all fixed points is closed The set $\left(A_{c} \cup\{0\}\right) \times[0,1]$ is closed by its definition (see (2.4)). From the lower a priori estimate $0<r \leqslant\|x\|$ it follows that the set $\operatorname{Fix}\left(\widehat{G}, A_{c} \times[0,1]\right)$ is closed, too. Its boundedness follows from the upper a priori estimate (see $(2.23)$ ). The completely continuous map $\widehat{G}$ sends bounded sets to relatively compact sets. Consequently, $\operatorname{Fix}\left(\widehat{G}, A_{c} \times[0,1]\right)$ is compact.

Proof of the lower and upper a priori estimate. Let $x=(u, v) \in A_{c}$ be a solution of the system $(2.20)$ for $\lambda \in[0,1]$. Observe that the kernels $\widehat{K}_{1}$ and $\widehat{K}_{1}$ are bounded independently of $\lambda \in[0,1]$ :

$$
\begin{gathered}
\left|\widetilde{K}_{i}(\ldots)\right|=\left|\lambda K_{i}(\ldots)+(1-\lambda) \delta_{i}\right|=\lambda\left|K_{i}(\ldots)\right|+(1-\lambda), \\
\underline{K} \leqslant\left|\widetilde{K}_{i}(\ldots)\right| \leqslant \bar{K} .
\end{gathered}
$$

We shall use the following notations:

$$
\begin{array}{ll}
\bar{u}=\max |u(t)|, \quad \underline{u}=\min |u(t)|, \\
\bar{v}=\max |v(t)|, \quad \underline{v}=\min |v(t)|,
\end{array}
$$

for $t \in[0,1]$, and

$$
A=\int_{0}^{1}|u(s)|^{\alpha} d s, \quad B=\int_{0}^{1}|v(s)|^{\beta} d s .
$$

From (2.27) and (2.26) we get

$$
\underline{u}^{\alpha} \leqslant A \leqslant \bar{u}^{\alpha}, \quad \underline{v}^{\beta} \leqslant B \leqslant \bar{v}^{\beta} .
$$

From (2.20), (2.24) and (2.27) we get

$$
\underline{K} B \leqslant \underline{u} \leqslant \bar{u} \leqslant \bar{K} B, \quad \underline{K} A \leqslant \underline{v} \leqslant \bar{v} \leqslant \bar{K} A .
$$

From (2.27) and (2.29) we get

$$
\begin{gathered}
(\underline{K} B)^{\alpha} \leqslant A \leqslant(\bar{K} B)^{\alpha}, \quad(\underline{K} A)^{\beta} \leqslant B \leqslant(\bar{K} A)^{\beta}, \\
\underline{K}^{\alpha(\beta+1)} A^{\alpha \beta} \leqslant A \leqslant \bar{K}^{\alpha(\beta+1)} A^{\alpha \beta}, \\
\underline{K}^{\beta(\alpha+1)} B^{\alpha \beta} \leqslant B \leqslant \bar{K}^{\beta(\alpha+1)} B^{\alpha \beta} .
\end{gathered}
$$

Case I) $0<\alpha \beta<1$. Then

$$
\begin{gathered}
\underline{K}^{\frac{\alpha(\beta+1)}{1-\alpha \beta}} \leqslant A \leqslant \bar{K}^{\frac{\alpha(\beta+1)}{1-\alpha \beta}}, \quad \underline{K}^{\frac{\beta(\alpha+1)}{1-\alpha \beta}} \leqslant B \leqslant \bar{K}^{\frac{\beta(\alpha+1)}{1-\alpha \beta}}, \\
\underline{K}^{\frac{\beta+1}{1-\alpha \beta}} \leqslant \bar{u} \leqslant \bar{K}^{\frac{\beta+1}{1-\alpha \beta}}, \quad \underline{K}^{\frac{\alpha+1}{1-\alpha \beta}} \leqslant \bar{v} \leqslant \bar{K}^{\frac{\alpha+1}{1-\alpha \beta}} .
\end{gathered}
$$

Case II) $1<\alpha \beta$. Then

$$
\begin{array}{cc}
\bar{K}^{\frac{\alpha(\beta+1)}{1-\alpha \beta}} \leqslant A \leqslant \underline{K}^{\frac{\alpha(\beta+1)}{1-\alpha \beta}}, \quad \bar{K}^{\frac{\beta(\alpha+1)}{1-\alpha \beta}} \leqslant B \leqslant \underline{K}^{\frac{\beta(\alpha+1)}{1-\alpha \beta}}, \\
\underline{K}_{\bar{K}}^{\frac{\beta(\alpha+1)}{1-\alpha \beta}} \leqslant \bar{u} \leqslant \bar{K} \underline{K}^{\frac{\beta(\alpha+1)}{1-\alpha \beta}}, \quad \underline{K} \bar{K}^{\frac{\alpha(\beta+1)}{1-\alpha \beta}} \leqslant \bar{v} \leqslant \bar{K} \underline{K}^{\frac{\alpha(\beta+1)}{1-\alpha \beta}} .
\end{array}
$$


The last two inequalities give lower and upper a priori estimates for $\|x\|$, where $\|x\|=$ $\bar{u}+\bar{v}$. Therefore, the compactness of $\operatorname{Fix}\left(\widehat{G}, A_{c} \times[0,1]\right)$ follows from Lemma 2.3.

We verified conditions (A)-(C) for the homotopy $x=G_{\lambda}(x)$ and so we have proved that this homotopy is admissible. Finally, we have to calculate the Nielsen number for the correspondence $G_{0}$.

Note that the image of $G_{0}: X \rightarrow X$ is the two-dimensional space of constant functions

$$
E^{2}=\left\{\left(c_{1}, c_{2}\right): c_{i} \in \mathbb{R}\right\}
$$

and thus all its fixed points belong to this plane. Moreover, $G_{0}\left(A_{c}\right) \subset E_{0}^{2}$, where $E_{0}^{2}$ is the punctured plane. The map $g$ defined by $(2.10)$ is the restriction of $G_{0}$ to the plane $E^{2}$. Finally, we have

$$
\mathbf{N}\left(G_{\lambda}, A_{c}\right)=\mathbf{N}\left(G_{0}, A_{c}\right)=\mathbf{N}\left(g, \mathbb{R}_{0}^{2}\right)
$$

and by Lemma 2.2 we know when this Nielsen number is non-zero.

3. Multidimensional system of integral equations. Consider a system of $2 n$ nonlinear integral equations of Urysohn type:

$$
\left\{\begin{array}{l}
u_{1}(t)=\int_{0}^{1} K_{11}(t, s, x(s)) v_{1}^{\beta_{1}}(s) d s \\
v_{1}(t)=\int_{0}^{1} K_{12}(t, s, x(s)) u_{1}^{\alpha_{1}}(s) d s \\
\cdots \\
u_{n}(t)=\int_{0}^{1} K_{n 1}(t, s, x(s)) v_{n}^{\beta_{n}}(s) d s \\
v_{n}(t)=\int_{0}^{1} K_{n 2}(t, s, x(s)) u_{n}^{\alpha_{n}}(s) d s
\end{array}\right.
$$

where $x=\left(u_{1}, v_{1}, \ldots, u_{n}, v_{n}\right) \in \mathbb{R}^{2 n}$.

We assume that the following conditions are satisfied for all $i=1, \ldots, n$ and $j=1,2$ :

1) $K_{i j}(t, s, x) \in C^{1}\left([0,1]^{2} \times \mathbb{R}^{2 n}\right)$;

2) $\underline{K}^{i j} \leqslant\left|K_{i j}(t, s, x)\right| \leqslant \bar{K}_{i j}$ for all $(t, s, x) \in[0,1]^{2} \times \mathbb{R}^{2 n}$,

where $0<\underline{K}^{i j} \leqslant 1 \leqslant \bar{K}_{i j}$;

3) $\alpha_{i}=n_{i 1} / m_{i 1}, \beta_{i}=n_{i 2} / m_{i 2} \in \mathbb{Q}_{+}$and $\alpha_{i} \beta_{i} \neq 1$.

We shall use the following notation:

$$
X=(C[0,1])^{2 n}, \quad A_{c}^{n}=A_{c} \times \ldots \times A_{c}, \quad\left(\mathbb{R}_{0}^{2}\right)^{n}=\mathbb{R}_{0}^{2} \times \ldots \times \mathbb{R}_{0}^{2} .
$$

The system (3.1) is equivalent to the operator equation $x=G(x)$, where the operator $G: X \rightarrow X$ is defined similarly as in (2.15). The map $G$ is completely continuous and $G\left(A_{c}^{n}\right) \subset A_{c}^{n}$. Note that the system $(3.1)$ has a trivial solution $x_{0}=(0, \ldots, 0)$.

THEOREM 3.1. Suppose that system (3.1) satisfies conditions 1-3 of (3.2). Then the set $\operatorname{Fix}\left(G, A_{c}^{n}\right)$ is compact. The Nielsen number $\mathbf{N}\left(G, A_{c}^{n}\right)$ is well defined and

$$
\mathbf{N}\left(G, A_{c}^{n}\right)=\mathbf{N}\left(g,\left(\mathbb{R}_{0}^{2}\right)^{n}\right)
$$


where

$$
g: \mathbb{R}_{0}^{2} \times \ldots \times \mathbb{R}_{0}^{2} \rightarrow \mathbb{R}_{0}^{2} \times \ldots \times \mathbb{R}_{0}^{2}
$$

is the map given by

$$
g\left(u_{1}, v_{1}, \ldots, u_{n}, v_{n}\right)=\left(\delta_{11} v_{1}^{\beta_{1}}, \delta_{12} u_{1}^{\alpha_{1}}, \ldots, \delta_{n 1} v_{n}^{\beta_{n}}, \delta_{n 2} u_{n}^{\alpha_{n}}\right)
$$

with $\delta_{i j}=\operatorname{sign} K_{i j}$ independent of $(t, s, x)$.

As in Theorem 2.1, the proof is based on the linear homotopy

$$
x=G_{\lambda}(x), \quad G_{\lambda}=\lambda G_{0}+(1-\lambda) G_{1}, \quad \lambda \in[0,1],
$$

to a simpler system

$$
\left\{\begin{array}{l}
u_{1}(t)=\int_{0}^{1} \delta_{11} v_{1}^{\beta_{1}}(s) d s \\
v_{1}(t)=\int_{0}^{1} \delta_{12} u_{1}^{\alpha_{1}}(s) d s \\
\cdots \\
u_{n}(t)=\int_{0}^{1} \delta_{n 1} v_{n}^{\beta_{n}}(s) d s \\
v_{n}(t)=\int_{0}^{1} \delta_{n 2} u_{n}^{\alpha_{n}}(s) d s
\end{array}\right.
$$

which is equivalent to the operator equation $x=G_{0}(x)$. Note that the corresponding operator $G_{0}: X \rightarrow X$ has a finite-dimensional image in the subspace of constant functions and its restriction is the map $g$ (see (3.5) and (3.6)). The technique of the proof of Theorem 3.1 is analogous to the proof of Theorem 2.1 .

Now we give an application of Theorem 3.1.

EXAmple 3.1. Consider a system of three pairs of nonlinear integral equations

$$
\left\{\begin{array}{l}
u_{1}(t)=\int_{0}^{1}\left(1+\sin ^{2}\left[t v_{1}^{3}(s)+u_{3}^{2}(s)\right]\right) v_{1}^{7}(s) d s \\
v_{1}(t)=\int_{0}^{1}\left(3+\cos \left[t u_{2}(s)\right]\right) u_{1}^{5}(s) d s \\
u_{2}(t)=\int_{0}^{1}\left(1+t^{2}+s^{4}\right) v_{2}^{3}(s) d s \\
v_{2}(t)=\int_{0}^{1}\left(3+t \sin \left[u_{2}^{4}(s)\right]\right) u_{2}^{5}(s) d s \\
u_{3}(t)=\int_{0}^{1} \ln (0.1+t s / 2) v_{3}^{9}(s) d s \\
v_{3}(t)=\int_{0}^{1} \arctan \left(2+u_{1}^{2}(s)+t^{3}+v_{3}^{4}(s)\right) u_{3}^{4}(s) d s
\end{array}\right.
$$

where $x=\left(u_{1}, v_{1}, u_{2}, v_{2}, u_{3}, v_{3}\right) \in \mathbb{R}^{6}$ and

$$
X=(C[0,1])^{6}, \quad A_{c}^{3}=A_{c} \times A_{c} \times A_{c}, \quad\left(\mathbb{R}_{0}^{2}\right)^{3}=\mathbb{R}_{0}^{2} \times \mathbb{R}_{0}^{2} \times \mathbb{R}_{0}^{2} .
$$


We reduce the system (3.9) to a finite-dimensional equation $x=g(x)$, where

$$
g: \mathbb{R}^{6} \rightarrow \mathbb{R}^{6}, \quad g\left(\left(\mathbb{R}_{0}^{2}\right)^{3}\right) \subset\left(\mathbb{R}_{0}^{2}\right)^{3},
$$

and $g$ is defined by the formula

$$
g\left(u_{1}, v_{1}, u_{2}, v_{2}, u_{3}, v_{3}\right)=\left(v_{1}^{7}, u_{1}^{5}, v_{2}^{3}, u_{2}^{5},-v_{3}^{9}, u_{3}^{4}\right) .
$$

The equation $x=g(x)$ has 4 solutions in $\left(\mathbb{R}_{0}^{2}\right)^{3}$ :

$$
\begin{aligned}
& x_{1}=(+1,+1,+1,+1,-1,+1), \\
& x_{2}=(-1,-1,+1,+1,-1,+1), \\
& x_{3}=(-1,-1,-1,-1,-1,+1), \\
& x_{4}=(+1,+1,-1,-1,-1,+1),
\end{aligned}
$$

which belong to different Nielsen classes.

Finally, we have a multiplicity result:

$$
\mathbf{N}\left(G, A_{c}^{n}\right)=\mathbf{N}\left(g,\left(\mathbb{R}_{0}^{2}\right)^{3}\right)=4
$$

yields that the system (3.9) has at least 4 non-zero solutions.

There is a direct approach to equations (2.1) and (3.1), based on the following theorem.

Theorem 3.2. Let the conditions (A)-(C) be satisfied. Assume that there exist subdomains $D_{j}(j=1, \ldots, n)$ in $D$ such that

$$
\begin{aligned}
& D_{i} \cap D_{j}=\emptyset, \quad i \neq j, \quad x_{j}^{0} \in D_{j}, \\
& G_{\lambda}\left(D_{j}\right) \subset D_{j}, \quad \operatorname{Fix}\left(G_{\lambda}, D\right) \cap \partial D_{j}=\emptyset,
\end{aligned}
$$

for all $j=1, \ldots, n$ and $\lambda \in[0,1]$. Then, the equation $x=G_{\lambda}(x)$ has at least one solution in each subdomain $D_{j}(j=1, \ldots, n)$ for each $\lambda \in[0,1]$.

The proof of Theorem 1.1 is based on the following property of degree:

$$
\operatorname{deg}\left(I-G_{\lambda}, D_{j}, 0\right)=\operatorname{deg}\left(I-G_{0}, U_{j}^{0}, 0\right) \neq 0 .
$$

REMARK 3.1. In the case of the system (2.1) the interior of the annulus $A_{c}$ may be written as a union of 4 open isolated cones, two of them invariant with respect to the operator $G$. In the case of the system (3.1) the interior of the annulus $A_{c}^{n}$ may be written as a union of $4^{n}$ open isolated cones, some of them invariant with respect to the operator $G$. The technique of a priori estimates and degree property (3.16) may be applied in every invariant cone independently.

\section{References}

[BKM1] A. Yu. Borisovich, Z. Kucharski and W. Marzantowicz, Nielsen number and lower estimate for the number of solutions to a certain system of nonlinear integral equations, in: Applied Aspects of Global Analysis. New Developments in Global Analysis series, Voronezh University Press, 1994, 3-11.

[BKM2] A. Yu. Borisovich, Z. KuCharski and W. Marzantowicz, Relative Nielsen number and a lower estimate of the number of components of an algebraic set, in: Global and 
Stochastic Analysis. New Developments in Global Analysis series, Voronezh University Press, 1995, 3-14.

[N1] J. NiELSEn, Über die Minimalzahl der Fixpunkte bei Abbildungstypen der Ringflächen, Math. Ann. 82 (1921), 83-93.

[N2] J. NiELSEN, Untersuchungen zur Topologie der geschlossenen zweiseitigen Flächen, I-III, Acta Math. 50, 53, 58 (1927, 1929, 1932), 189-358, 1-76, 87-167.

[W] F. Wecken, Fixpunktklassen, I-III, Math. Ann. 117, 118, 118 (1941, 1942, 1942), 659-671, 216-234, 544-577.

[J] B. J. JIAng, Lectures on Nielsen Fixed Point Theory, Contemp. Math. 14 (1983).

[Br1] R. BRown, A topological bound on the number of distinct zeros of an analytic function, Pacific J. Math. 118 (1983), 53-58.

[Br2] R. Brown, Nielsen fixed point theory and parametrized differential equations, Contemp. Math. 72 (1988), 33-46.

[Br3] R. Brown, Retraction methods in the Nielsen fixed point theory, Pacific J. Math. 115 (1984), 277-297.

[Br4] R. Brown, The Lefschetz Fixed Point Theorem, Chicago, 1972.

[Sch] H. Schirmer, A relative Nielsen number, Pacific J. Math. 122 (1986), 253-266.

[K] Kiang Tsai-han, The Theory of Fixed Point Classes, Springer, Berlin, 1989.

[S] K. Scholz, The Nielsen fixed point theory for non-compact spaces, Rocky Mountain J. Math. 4 (1974), 81-87.

[F] M. FEČKAN, Nielsen fixed point theory and nonlinear equations, J. Differential Equations 106 (1993), 312-331. 